\title{
Lösliche Lanthanid-alkoxide mit niedrigen Koordinationszahlen am Metall-Atom
}

\author{
Michael Wedler, John W. Gilje, Ursula Pieper, Dietmar Stalke, Mathias Noltemeyer und Frank T. Edelmann*
}

Institut für Anorganische Chemie der Universität Göttingen, Tammannstr. 4, D-3400 Göttingen

Eingegangen am 12. Juli 1990

Key Words: Lanthanide alkoxides / Tritox ligand / Neodymium

\section{Soluble Lanthanide Alkoxides with Low Coordination Numbers at the Metal Atom}

$\mathrm{Nd}\left[\mathrm{N}\left(\mathrm{SiMe}_{3}\right)_{2} \mathrm{l}_{3}(\text { thf })_{2}\right.$ (1) reacts with $t \mathrm{Bu}_{3} \mathrm{COH}$ (2) to give the monomeric lanthanide alkoxide (tritox) ${ }_{3} \mathrm{Nd}$ (thf) (3). The chloro-bridged dimer $\left[(\text { tritox })_{2} \mathrm{Nd}(\mu-\mathrm{Cl}) \text { thf }\right]_{2}(6)$ is obtained by treatment of $\mathrm{NdCl}_{3}(\text { thf })_{2}$ (4) with $\mathrm{LiOC} \mathrm{Bu}_{3}$ (5). The structure of 6 has been determined by X-ray crystallography.
Wohldefinierte Metallalkoxide sind von Bedeutung als Vorstufen für reinste Metalloxide. Zur Umwandlung von Alkoxiden in die entsprechenden Metalloxide dienen in erster Linie Thermolyseverfahren wie die Metal-Organic-Chemical-Vapor-Deposition(MOCVD-)Technik sowie der Sol-Gel-Prozess". Auch von den Lanthanid-Elementen (einschließlich Sc, Y und La) sind zahlreiche Alkoxide und Phenolate bekannt ${ }^{2)}$. Einfache Lanthanid(III)-phenolate bilden in der Regel unlösliche Produkte. Monomere, homoleptische Derivate lassen sich in bewährter Weise durch Einführung sperriger Phenolat-Liganden wie 2,6-t $\mathrm{Bu}_{2}-4-\mathrm{X}-\mathrm{C}_{6} \mathrm{H}_{2} \mathrm{O}^{\ominus}(\mathrm{X}=$ $\mathrm{H}, \mathrm{Me}$ ) gewinnen ${ }^{3-5)}$. Lanthanid(III)-alkoxide liegen sowohl in Lösung als auch im Festkörper stets als Oligomere vor. $\mathrm{Zu}$ den wenigen strukturell gesicherten Beispielen gehört das „Neodym(III)isopropylat" $\mathrm{Nd}_{6}\left(\mu_{6}-\mathrm{Cl}\right)\left(\mu_{3}-\mathrm{O} i \mathrm{Pr}\right)_{2}(\mu-\mathrm{O} i \mathrm{Pr})_{9}(\mathrm{O} i \mathrm{Pr})_{6}{ }^{6}$. Eine Rcihe von Yttrium(III)-alkoxiden leitet sich von der dreikernigen Einheit $Y_{3}\left(\mu_{3}-\mathrm{OR}\right)\left(\mu_{3}-\mathrm{X}\right)(\mu-\mathrm{OR})_{3}(\mathrm{X}=\mathrm{O}, \mathrm{OR}, \mathrm{Cl}) \mathrm{ab}^{7}$. Wir berichten hier über die Verwendung des raumerfüllenden $t \mathrm{Bu}_{3} \mathrm{CO}^{\ominus}$-(,tritox“-)Liganden $^{5-10)}$ zur Synthese einfacher Lanthanid(III)-alkoxide mit niedrigen Koordinationszahlen $(\leq 5)$ am Metall-Atom ${ }^{11)}$.

Die Reaktion von $\mathrm{Nd}\left[\mathrm{N}\left(\mathrm{SiMe}_{3}\right)_{2}\right]_{3}(\text { thf })_{2}(1)$ mit drei Äquivalenten $t \mathrm{Bu}_{3} \mathrm{COH}$ (2) liefert das monomere Neodym(III)-alkoxid (tritox) ${ }_{3} \mathrm{Nd}$ (thf) (3) mit $88 \%$ Ausbeute. Die himmelblauen Kristalle von 3 schmelzen bei $172^{\circ} \mathrm{C}$ und sind auch in unpolaren Lösungsmitteln wie Hexan leicht löslich. Zwei stark hochfeldverschobene ${ }^{1} \mathrm{H}$-NMR-Signale bei $\delta=-22.73$ und -46.14 zeigen die Anwesenheit eines koordinierten THF-Moleküls an. Einkristalle für eine Röntgenstrukturanalyse ${ }^{12)}$ erhält man aus Hexan. Es liegt ein monomeres Lanthanid-alkoxid vor, in dem das Neodym-Zentralatom verzerrt tetraedrisch von vier Sauerstoff-Atomen umgeben ist.

$$
\underset{1}{\mathrm{Nd}\left[\mathrm{N}\left(\mathrm{SiMe}_{3}\right)_{2}\right]_{3}\left(\mathrm{thf}_{2}+3 t \mathrm{Bu}_{3} \mathrm{COH}\right.} \stackrel{\text { Hexan }}{\stackrel{\text { (tritox })_{3} \mathrm{Nd}(\text { thf })+3 \mathrm{NH}\left(\mathrm{SiMe}_{3}\right)_{2}}{\longrightarrow}}
$$$$
3
$$

Anders verläuft die Umsetzung von $\mathrm{NdCl}_{3}\left(\mathrm{thf}_{2}\right.$ (4) mit $\mathrm{LiOCtBu}$ (5) im Molverhältnis 1:2. Hier erhält man zunächst ein schwerlösliches, hellblaues Produkt der analytischen Zusammensetzung $\mathrm{Li}\left[(\text { tritox })_{2} \mathrm{NdCl}_{2}(\text { thf })_{2}\right]$. Durch eine modifizierte ,Toluol-RückflußMethode“ ${ }^{13)}$ läßt sich das koordinierte Lithiumchlorid entfernen, und man erhält in guter Ausbeute (71\%) das dimere Chloroalkoxid $\left[(\text { tritox })_{2} \mathrm{Nd}(\mu-\mathrm{Cl}) \text { thf }\right]_{2}$ (6).

$$
2 \mathrm{NdCl}_{3}\left(\text { thf }_{2}+4 \mathrm{LiOCtBu} 3 \frac{\text { 1.THF, 2. Toluol }}{-4 \mathrm{LiCl},-2 \mathrm{THF}}\right.
$$

4

5

$\left[(\text { tritox })_{2} \mathrm{Nd}(\mu-\mathrm{Cl}) \text { thf }\right]_{2}$ 6

Durch Umkristallisieren aus Toluol werden große, blauviolette Kristalle erhalten. ${ }^{1} \mathrm{H}$-NMR-Signale bei $\delta=-20.47$ und -44.55 sind wiederum koordiniertem THF zuzuordnen. Die Röntgenstrukturanalyse (Abb. 1, Tab. 1) zeigt das Vorliegen eines über $\mathrm{Cl}$ verbrückten dimeren Neodym(III)-alkoxids. Die asymmetrische Einheit enthält zwei strukturell nahezu gleiche Moleküle. Beide besitzen ein Inversionszentrum in der Mitte der $\mathrm{Nd}-\mathrm{Nd}$-Verbindungsachse. Die Neodym-Atome sind nunmehr fünffach koordiniert. Der zentrale viergliedrige $\mathrm{Nd}_{2} \mathrm{Cl}_{2}$ - Ring ist planar und besitzt alternierende $\mathrm{Nd}-\mathrm{Cl}$-Bindungslängen. Bindungslängen und -winkel in der $\mathrm{Nd}_{2} \mathrm{Cl}_{2}$-Einheit ähneln denen im ebenfalls über Chlor-Atome verbrückten $\left[\eta-1,3-\left(\mathrm{Me}_{3} \mathrm{Si}\right)_{2} \mathrm{C}_{5} \mathrm{H}_{3}\right]_{2} \mathrm{Nd}(\mu-\mathrm{Cl})_{2} \mathrm{Li}(\operatorname{thf})_{2}{ }^{14)}$.

Der tritox-Ligand wurde von Wolczanski et al. als sterisches Cyclopentadienyl- Äquivalent in die Übergangsmetallchemie ein-

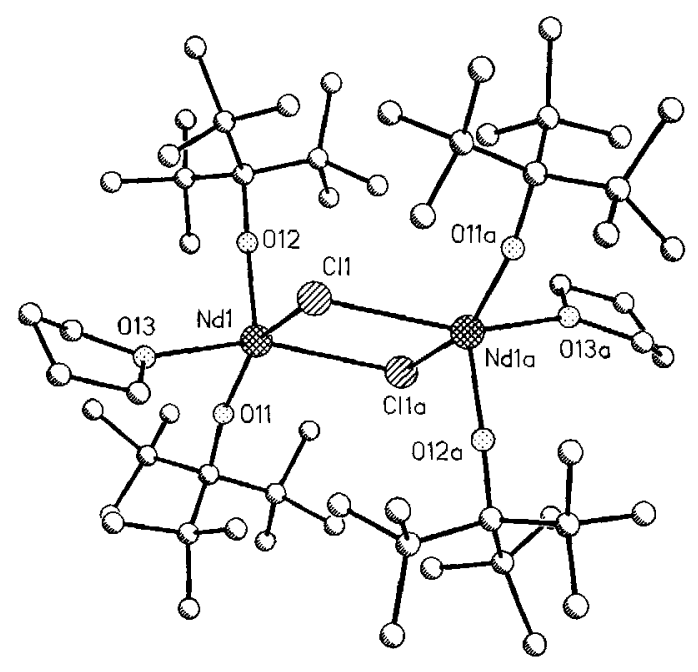

Abb. 1. Struktur von $6 \mathrm{im}$ Kristall (eines von zwei kristallographisch unabhängigen Molekülen) 
geführt ${ }^{5}$. Auch bei den hier beschriebenen Lanthanid-alkoxiden zcigt sich eine erstaunliche strukturelle Analogie zwischen tritoxund Cyclopentadienyl-Derivaten. Den Titelverbindungen 3 und 6 entsprechen die Cyclopentadienyl-Komplexe $\left(\mathrm{C}_{5} \mathrm{H}_{5}\right)_{3} \mathrm{Nd}(\mathrm{thf})^{15)}$ und $\left[\left(\mathrm{C}_{5} \mathrm{H}_{5}\right)_{2} \mathrm{Nd}(\mu-\mathrm{Cl}) \text { thf }\right]_{2}{ }^{16)}$. So sind ctwa die $\mathrm{O}$ (tritox)- $\mathrm{Nd}-$ $\mathrm{O}$ (tritox)-Winkel in $3\left(116.5,117.5\right.$ und $\left.120.1^{\circ}\right)$ nahezu gleich den entsprechenden Winkeln zwischen dem Neodym-Atom und den Cyclopentadienyl-Ringmittelpunkten in $\left(\mathrm{C}_{5} \mathrm{H}_{5}\right)_{3} \mathrm{Nd}$ (thf) $(115.0,117.1$ und $\left.120.5^{\circ}\right)^{15}$.

Die vorliegenden Ergebnisse zeigen, daß der tritox-Ligand vorzüglich geeignet ist, nicht nur nicdrige Koordinationszahlen an Übergangsmetallen ${ }^{8-10)}$ sondern auch an Lanthaniden zu stabili-

Tab. 1. Ausgewählte Bindungsabstände [pm] und Winkel ["] von 6

\section{Molekül 1}

$\mathrm{Nd}(1)-\mathrm{Cl}(1) \quad 288.3$ (1)

$\mathrm{Nd}(1)-\mathrm{Cl}$ (1a) 280.8 (1)

$\mathrm{Nd}(1)-0(11) \quad 210.9$ (2)

$\operatorname{Nd}(1)-0(12) \quad 209.0$ (3)

$\mathrm{Nd}(1)-0(13) 252.9$ (2)

$\begin{array}{lr}\mathrm{C} 1(1)-\mathrm{Nd}(1)-\mathrm{C} 1(1 \mathrm{a}) & 74.8(1) \\ \mathrm{Nd}(1)-0(11)-\mathrm{C}(11) & 163.8(2) \\ \mathrm{Nd}(1)-\mathrm{O}(12)-\mathrm{C}(120) & 174.4(2) \\ O(11)-\mathrm{Nd}(1)-O(12) & 108.9(1) \\ O(11)-\mathrm{Nd}(1)-0(13) & 93.0(1) \\ O(12)-\mathrm{Nd}(1)-O(13) & 106.0(1)\end{array}$

\section{Molekül 2 \\ Nd(2)-C1(2) 281.7 (1) \\ $\mathrm{Nd}(2)-\mathrm{C1}$ (2a) 286.2 (1) \\ $\mathrm{Nd}(2)-\mathrm{O}(21) \quad 210.8$ (2) \\ $\mathrm{Nd}(2)-\mathrm{O}(22) \quad 211.4$ (3) \\ $\mathrm{Nd}(2)-\mathrm{O}(23) \quad 252.1$ (2)}

$\mathrm{Cl}(2)-\mathrm{Nd}(2)-\mathrm{Cl}(2 \mathrm{a}) \quad 74.0(1)$ $\mathrm{Nd}(2)-\mathrm{O}(21)-\mathrm{C}(21) \quad 166.2(2)$ $\mathrm{Nd}(2)-\mathrm{O}(22)-\mathrm{C}(220) \quad 168.3(2)$ $\mathrm{O}(21)-\mathrm{Nd}(2)-\mathrm{O}(22) \quad 111.4(1)$ $\mathrm{O}(21)-\mathrm{Nd}(2)-\mathrm{O}(23) \quad 100.3(1)$ $O(22)-N d(2)-O(23) \quad 94.8(1)$

Tab. 2. Atomkoordinaten $\left(\times 10^{4}\right)$ und äquivalente isotrope Thermalparameter $\left(\times 10^{-1}\right)\left[\mathrm{pm}^{3}\right]$ von 6; äquivalente isotrope $U$ berechnet als cin Drittel der Spur des orthogonalen $U_{i, j}$-Tensors

\begin{tabular}{|c|c|c|c|c|}
\hline & $\mathrm{x}$ & $\mathrm{y}$ & $\mathbf{z}$ & $\mathrm{U}(\mathrm{eq})$ \\
\hline \multicolumn{5}{|c|}{ Molekü1 1} \\
\hline $\operatorname{Nd}(1)$ & $6126(1)$ & $3573(1)$ & $4703(1)$ & $20(1)$ \\
\hline $\mathrm{Cl}(1)$ & $4601(1)$ & $4349(1)$ & $5665(1)$ & $29(1)$ \\
\hline $0(11)$ & $7632(2)$ & $3307(2)$ & $4259(1)$ & $28(1)$ \\
\hline $0(12)$ & $5221(2)$ & $2959(2)$ & $4235(1)$ & $27(1)$ \\
\hline $0(13)$ & $6787(2)$ & $2306(2)$ & $5641(1)$ & $35(1)$ \\
\hline$C(11)$ & $8679(3)$ & $3300(3)$ & $4084(2)$ & $33(1)$ \\
\hline$C(12)$ & $8601(3)$ & $4287(3)$ & $3576(2)$ & $38(2)$ \\
\hline$C(13)$ & $9617(3)$ & $4277(4)$ & $3173(2)$ & $53(2)$ \\
\hline$C(14)$ & $8269(3)$ & $5295(3)$ & $3853(2)$ & $46(2)$ \\
\hline$C(15)$ & $7701(3)$ & $4460(3)$ & $3120(2)$ & $47(2)$ \\
\hline$C(16)$ & $9236(3)$ & $2251(3)$ & $3793(2)$ & $37(2)$ \\
\hline$C(17)$ & $8880(3)$ & $2257(3)$ & $3135(2)$ & $44(2)$ \\
\hline$C(18)$ & $8904(3)$ & $1359(3)$ & $4169(2)$ & $45(2)$ \\
\hline$C(19)$ & $10448(3)$ & $1926(3)$ & $3742(2)$ & $52(2)$ \\
\hline$c(110)$ & $9196(3)$ & $3363(3)$ & $4724(2)$ & $38(2)$ \\
\hline $\mathrm{C}(111)$ & $10208(3)$ & $3668(4)$ & $4638(2)$ & $53(2)$ \\
\hline$c(112)$ & $9449(3)$ & $2365(3)$ & $5171(2)$ & $48(2)$ \\
\hline$C(113)$ & $8397(3)$ & $4092(3)$ & $5130(2)$ & $41(2)$ \\
\hline$C(120)$ & $4533(3)$ & $2570(3)$ & $3965(2)$ & $28(1)$ \\
\hline$C(121)$ & $5294(3)$ & $1629(3)$ & $3622(2)$ & $34(1)$ \\
\hline$c(122)$ & $5799(3)$ & $702(3)$ & $4086(2)$ & $52(2)$ \\
\hline$G(123)$ & $6260(3)$ & $1883(3)$ & $3288(2)$ & $41(2)$ \\
\hline$C(124)$ & $4750(3)$ & $1266(3)$ & $3144(2)$ & $48(2)$ \\
\hline$C(125)$ & $3860(3)$ & $3488(3)$ & $3469(2)$ & $34(1)$ \\
\hline$c(128)$ & $3545(3)$ & $4501(3)$ & $3741(2)$ & $46(2)$ \\
\hline$c(126)$ & $4500(3)$ & $3713(3)$ & $2874(2)$ & $46(2)$ \\
\hline$C(127)$ & $2861(3)$ & $3337(3)$ & $3253(2)$ & $44(2)$ \\
\hline$C(129)$ & $3812(3)$ & $2228(3)$ & $4542(2)$ & $35(2)$ \\
\hline$C(130)$ & $4444(3)$ & $1744(3)$ & $5126(2)$ & $48(2)$ \\
\hline$C(131)$ & $2964(3)$ & $3116(3)$ & $4791(2)$ & $45(2)$ \\
\hline$C(132)$ & $3226(3)$ & $1496(3)$ & $4403(2)$ & $51(2)$ \\
\hline$C(140)$ & $7104(3)$ & $1224(3)$ & $5655(2)$ & $45(2)$ \\
\hline$C(141)$ & $6867(8)$ & $830(6)$ & $6288(3)$ & $54(4)$ \\
\hline$C\left(141^{\prime}\right)$ & $7638(10)$ & $808(9)$ & $6266(5)$ & $47(5)$ \\
\hline$C(142)$ & $7059(4)$ & $1556(3)$ & $6689(2)$ & $62(2)$ \\
\hline$C(143)$ & $6868(4)$ & $2526(3)$ & $6272(2)$ & $47(2)$ \\
\hline
\end{tabular}

Tab. 2 (Fortsetzung)

\begin{tabular}{|c|c|c|c|c|}
\hline & $\mathbf{x}$ & $y$ & $z$ & $\mathrm{U}(\mathbf{e q})$ \\
\hline \multicolumn{5}{|c|}{ Molekül 2} \\
\hline $\mathrm{Nd}(2)$ & $9966(1)$ & $-1525(1)$ & $-196(1)$ & $18(1)$ \\
\hline $\mathrm{Cl}(2)$ & $9967(1)$ & $395(1)$ & $-783(1)$ & $27(1)$ \\
\hline$O(21)$ & $11469(2)$ & $-2471(2)$ & $-414(1)$ & $25(1)$ \\
\hline $0(22)$ & $8816(2)$ & $-1696(2)$ & $-744(1)$ & $23(1)$ \\
\hline$O(23)$ & $9469(2)$ & $-2543(2)$ & $771(1)$ & $29(1)$ \\
\hline$c(21)$ & $12536(3)$ & $-2886(2)$ & $-587(2)$ & $24(1)$ \\
\hline$C(22)$ & $13175(3)$ & $-3072(3)$ & $48(2)$ & $29(1)$ \\
\hline$c(23)$ & $12686(3)$ & $-2220(3)$ & $453(2)$ & $42(2)$ \\
\hline$c(24)$ & $13106(3)$ & $-4001(3)$ & $502(2)$ & $44(2)$ \\
\hline$c(25)$ & $14356(3)$ & $-3184(3)$ & $-49(2)$ & $44(2)$ \\
\hline$C(26)$ & $12829(3)$ & $-2056(3)$ & $-1117(2)$ & $29(1)$ \\
\hline$C(27)$ & $11962(3)$ & $-1585(3)$ & $-1600(2)$ & $41(2)$ \\
\hline$c(28)$ & $12901(3)$ & $-1123(3)$ & $-851(2)$ & $39(2)$ \\
\hline $\mathrm{C}(29)$ & $13873(3)$ & $-2442(3)$ & $-1496(2)$ & $44(2)$ \\
\hline$C(210)$ & 12615 (3) & $-3920(3)$ & $-860(2)$ & $34(1)$ \\
\hline$C(211)$ & $13742(3)$ & $-4657(3)$ & $-914(2)$ & $46(2)$ \\
\hline$G(212)$ & $12163(3)$ & $-3755(3)$ & $-1508(2)$ & $46(2)$ \\
\hline$C(213)$ & $11943(3)$ & $-4516(3)$ & $-465(2)$ & $43(2)$ \\
\hline$C(220)$ & $7901(3)$ & $-1697(3)$ & $-1024(2)$ & $22(1)$ \\
\hline$C(221)$ & $6966(3)$ & $-1445(3)$ & $-483(2)$ & $28(1)$ \\
\hline$C(222)$ & $5835(3)$ & $-1092(3)$ & $-718(2)$ & $41(2)$ \\
\hline$c(223)$ & $6999(3)$ & $-2339(3)$ & $31(2)$ & $37(2)$ \\
\hline$c(224)$ & $7083(3)$ & $-643(3)$ & $-108(2)$ & $36(2)$ \\
\hline$C(225)$ & $7736(3)$ & $-826(3)$ & $-1617(2)$ & $27(1)$ \\
\hline$C(226)$ & $7345(3)$ & $249(3)$ & $-1439(2)$ & $35(1)$ \\
\hline$c(227)$ & $8780(3)$ & $-826(3)$ & $-1978(2)$ & $38(2)$ \\
\hline$C(228)$ & $6957(3)$ & $-865(3)$ & $-2094(2)$ & $40(2)$ \\
\hline$C(229)$ & $8123(3)$ & $-2811(3)$ & $-1226(2)$ & $29(1)$ \\
\hline$C(230)$ & $7140(3)$ & $-3103(3)$ & $-1349(2)$ & $42(2)$ \\
\hline$C(231)$ & $8857(3)$ & $-3002(3)$ & $-1815(2)$ & $41(2)$ \\
\hline$c(232)$ & $8760(3)$ & $-3632(3)$ & $-73 I(2)$ & $40(2)$ \\
\hline$C(240)$ & $9874(3)$ & $-3626(3)$ & $880(2)$ & $45(2)$ \\
\hline$C(241)$ & $9508(4)$ & $-3961(3)$ & $1512(2)$ & $52(2)$ \\
\hline$C(242)$ & $8566(3)$ & $-3113(3)$ & $1655(2)$ & $53(2)$ \\
\hline$c(243)$ & $8807(3)$ & $-2190(3)$ & $1312(2)$ & $39(2)$ \\
\hline \multicolumn{5}{|c|}{ Moleküle 3 und 4 (Toluol) } \\
\hline$C(50)$ & $995(8)$ & $1148(7)$ & $6896(5)$ & $210(7)$ \\
\hline $\mathrm{C}(51)$ & $104(8)$ & $1946(6)$ & $7096(5)$ & $130(5)$ \\
\hline$c(52)$ & $-496(10)$ & $1677(8)$ & $7553(4)$ & $158(8)$ \\
\hline$c(53)$ & $-1363(11)$ & 2410 (14) & $7787(5)$ & 214 (13) \\
\hline$c(54)$ & $-1576(7)$ & $3342(8)$ & $7526(3)$ & $124(5)$ \\
\hline$C(55)$ & $-943(7)$ & $3618(5)$ & $7042(3)$ & $99(4)$ \\
\hline$c(56)$ & $-88(6)$ & $2905(5)$ & $6835(3)$ & $78(3)$ \\
\hline$C(60)$ & $4934(5)$ & $3631(5)$ & $7683(3)$ & $114(4)$ \\
\hline$c(61)$ & $4514(4)$ & $2769(4)$ & $7683(2)$ & $61(2)$ \\
\hline$C(62)$ & $4503(4)$ & $2097(5)$ & $8209(3)$ & $79(3)$ \\
\hline$c(63)$ & $4098(4)$ & $1320(5)$ & $8205(3)$ & $83(3)$ \\
\hline$C(64)$ & $3707(5)$ & $1178(4)$ & $7687(3)$ & $83(3)$ \\
\hline$C(65)$ & $3701(4)$ & $1830(5)$ & $7153(3)$ & $80(3)$ \\
\hline $\mathrm{C}(66)$ & $4103(4)$ & $2645(4)$ & $7158(2)$ & $66(2)$ \\
\hline
\end{tabular}

sieren. Mit 6 steht erstmals ein einfaches funktionalisierbares Lanthanid(III)-alkoxid zur Verfügung, das eine interessante Folgechemie ermöglichen solite.

Wir danken Herrn Prof. Dr. Herbert W. Roesky und Herrn Prof. George M. Sheldrick für die großzügige Unterstützung dieser Arbeit und Frau Dr. Sally Brooker für wertvolle Diskussionsbeiträge. F. T. E. ist der Deutschen Forschungsgemeinschaft für die Gewährung eines Habilitationsstipendiums zu Dank verpflichtet. J. W. G. dankt der Alexander-von-Humboldt-Stiftung für die Zuerkennung eines Humboldt-Forschungspreises.

\section{Experimenteller Teil}

(Tetrahydrofuran) tris (tri-tert-butylmethoxy) neodym(III) (3): Zu einer Lösung von $2.50 \mathrm{~g}(3.3 \mathrm{mmol}) \mathrm{Nd}\left[\mathrm{N}\left(\mathrm{SiMe}_{3}\right)_{2}\right]_{3}(\text { thf })_{2}{ }_{2}^{17)}$ in $50 \mathrm{ml}$ Hexan werden unter $\mathrm{N}_{2} 1.95 \mathrm{~g}(9.8 \mathrm{mmol}) t \mathrm{Bu}_{3} \mathrm{COH}^{18}$ in $20 \mathrm{ml}$ Hexan getropft. Nach $15 \mathrm{stdg}$. Rühren bei Raumtemp. wird durch eine dünne Schicht Celite filtriert und das Filtrat auf $20 \mathrm{ml}$ einge- 
engt. Bei $-25^{\circ} \mathrm{C}$ bilden sich himmelblaue Kristalle von 3; Ausb. $2.32 \mathrm{~g}(83 \%)$, Schmp. $172^{\circ} \mathrm{C}$. - IR (Nujol): $\tilde{v}=1247 \mathrm{~cm}^{-1}$ sst, 979 st, 941 st, 844 sst, 757 st. - ${ }^{1} \mathrm{H}-\mathrm{NMR}\left(80 \mathrm{MHz}, \mathrm{C}_{6} \mathrm{D}_{6}, 32^{\circ} \mathrm{C}\right): \delta=$ $6.46(\mathrm{~s}, 81 \mathrm{H}, t \mathrm{Bu}),-22.73(\mathrm{~m}, 4 \mathrm{H}, \mathrm{THF}),-46.14(\mathrm{~m}, 4 \mathrm{H}, \mathrm{THF})$.

\section{$\mathrm{C}_{43} \mathrm{H}_{89} \mathrm{NdO}_{4}$ (841.4) Ber. C $63.4 \mathrm{H} 11.0$ Gef. C $63.2 \mathrm{H} 11.2$}

Bis/ $\mu$-chloro(tetrahydrofuran) bis (tri-tert-butylmethoxy) neodym(III)] (6): $\mathrm{Zu}$ eincr Lösung von $2.96 \mathrm{~g}(7.50 \mathrm{mmol}) \mathrm{NdCl}_{3}(\text { thf })_{2}{ }^{19)}$ in $70 \mathrm{ml}$ THF tropft man unter $\mathrm{N}_{2} 3.09 \mathrm{~g}(15.0 \mathrm{mmol}) \mathrm{LiOC} t \mathrm{Bu}_{3}{ }^{8,10}$ in $30 \mathrm{ml}$ THF. Nach 15stdg. Rühren bei Raumtemp. wird zur Trockne eingedampft. Der Rückstand wird auf eine Fritte gegeben und mit ca. $100 \mathrm{ml}$ siedendem Toluol so lange extrahiert, bis das Filtrat farblos abläuft. Die Extraktionslösung wird durch eine dünne Schicht Celite filtriert. Kristallisation bei $0^{\circ} \mathrm{C}$ liefert $3.46 \mathrm{~g}$ (71\%) großc, blauviolette Kristalle. Beim Trocknen i. Vak. verliert die Substanz das locker gebundene Kristall-Toluol und geht in ein himmelblaues Pulver über, Schmp. $175^{\circ} \mathrm{C}$ (Zers.). - IR (Nujol): $\tilde{v}=1183 \mathrm{~cm}^{-1}$ st, 1055 sst, $1033 \mathrm{st}, 992$ sst, 925 st, $886 \mathrm{st}, 671$ sst. $-{ }^{1} \mathrm{H}-\mathrm{NMR}\left(80 \mathrm{MHz}, \mathrm{C}_{6} \mathrm{D}_{6}, 32^{\circ} \mathrm{C}\right): \delta=12.10\left(\mathrm{v}_{1 / 2}=40 \mathrm{~Hz}\right.$, $108 \mathrm{H}, t \mathrm{Bu}),-20.47(\mathrm{~m}, 8 \mathrm{H}, \mathrm{THF}),-44.55(\mathrm{~m}, 8 \mathrm{H}, \mathrm{THF})$.

$$
\mathrm{C}_{60} \mathrm{H}_{124} \mathrm{Cl}_{2} \mathrm{Nd}_{2} \mathrm{O}_{6}(1301.0) \quad \text { Ber. C } 55.4 \mathrm{H} 9.6 \mathrm{Cl} 5.4
$$$$
\text { Gef. C } 55.4 \text { H } 9.9 \mathrm{Cl} 4.9
$$

Kristallstrukturanalyse von $6^{20)}: \mathrm{C}_{60} \mathrm{H}_{124} \mathrm{Cl}_{2} \mathrm{Nd}_{2} \mathrm{O}_{6} \cdot 2 \mathrm{C}_{7} \mathrm{H}_{8}\left(M_{\mathrm{T}}=\right.$ 1485.2); Raumgruppe $P \overline{1} ; a=1338.8(3), b=1410.4(4), c=$ $2171.8(5) \mathrm{pm} ; \alpha=81.46(1), \beta=85.01(1), \gamma=72.28(2)^{\circ} ; \quad V=$ $3.8592 \mathrm{~nm}^{3} ; Z=2 ; \mu\left(\mathrm{Mo}-K_{\alpha}\right)=1.450 \mathrm{~mm}^{-1} ;$ Kristallgröße $0.5 \times$ $0.5 \times 0.6 \mathrm{~mm} ; \quad T=-120^{\circ} \mathrm{C}$; STOE-Vierkreisdiffraktometer; max./min. Transmission $0.895 / 0.836 ; 12854$ Reflexe bis $2 \theta_{\max }=45^{\circ}$ gemessen, davon 9890 symmetrieunabhängig und 8937 mit $F_{\mathrm{o}}>$ $4 \sigma\left(F_{0}\right)$ beobachtet; semiempirische Absorptionskorrektur; Strukturlösung und -verfeinerung mit SHELXS-86; 766 verfeinerte Parameter; $R=0.027 ; R_{w}=0.033, w^{-1}=\sigma^{2}\left(F_{\mathrm{o}}\right)+0.0001 \cdot F_{0}^{2}$. Atomkoordinaten und Thermalparameter finden sich in Tab. 2.

\section{CAS Registry Numbers}

1: $132622-49-2$ / 3: $132622-50-5$ / 6: 132622-51-6 / $\mathrm{NdCl}_{3}(\text { thf })_{2}$ : $126184-13-2$

\footnotetext{
1) Übersichten: ${ }^{\text {a) }}$ L. G. Hubert-Pfalzgraf, New J. Chem. 11 (1987) 663. - ${ }^{1 b)}$ D. C. Bradley, Chem. Rev. 89 (1989) 1317.
}

2) 2a) D. C. Bradley, R. C. Mehrotra, D. P. Gaur, Metal Alkoxides, Academic Press, London 1978. - ${ }^{26)}$ D. C. Bradley, Adv. Inorg. Chem. Radiochem. 15 (1972) 259.

3) 3al P. B.Hitchcock, M. F. Lappert. A. J. Singh, J. Chem. Soc., Chem. Commun. 1983, 1499. - ${ }^{\text {3b) }}$ P. B. Hitchcock, M. F. Lappert, R. G. Smith, Inorg. Chim. Acta 139 (1987) 183.

4) W. J. Evans, J. M. Olofson, J. W. Ziller, Inorg. Chem. 28 (1989)

${ }^{5)}{ }^{43 i}$ H. E. Stecher, A. Sen, A. L. Rheingold, Inorg, Chem. 27 (1988) 1130. - ${ }^{5 b}$ H. J. Heeres, A. Meetsma, J. H. Teuben, R. D. Rogers, Organometallics 8 (1989) 2637.

${ }^{6}$ R. A. Andersen, D. H. Templeton, A. Zalkin, Inorg. Chem. 17 (1978) 1962.

7) J. M. Olofson, R. E. Golden, J. W. Ziller, W. J. Evans, 44th ACS Northwest Regional Meeting, Reno, Nevada, 14. bis 16. Juni 1989.

8) T. V. Lubben, P. T. Wolczanski, G. D. Van Duyne, Organometallics 3 (1984) 977.

9) J. Hvoslef, H. Hope, B. D. Murray, P. P. Power, J. Chem. Soc., Chem. Commun. 1983, 1438.

10) G. Beck, P. B. Hitchcock, M. F. Lappert, I. A. MacKinnon, J. Chem. Soc., Chem. Commun. 1989, 1312.

11) (tritox) $)_{3} \mathrm{Ce}$ wurde kürzlich erwähnt, aber nicht charakterisiert: H. E. Stecher, A. Sen, A. L. Rheingold, Inorg. Chem. 28 (1989) 3280 .

12) Kristalldaten von $3^{20)}: \mathrm{C}_{43} \mathrm{H}_{83} \mathrm{O}_{4} \mathrm{Nd}\left(M_{\mathrm{r}}=808.3\right.$ ); Raumgruppe $P \overline{1} ; a=1220.1(4), b=1316.5(5), c=1693.6(6) \mathrm{pm} ; \alpha=$ $69.88(2), \beta=87.62(2), \gamma=62.34(1)^{\circ}, Z=2$. Die Datensammlung erfolgte bei $T=20^{\circ} \mathrm{C}$. Die Kristallstrukturanalyse bestätigt eindeutig das Vorliegen der monomeren Verbindung (tritox) ${ }_{3} \mathrm{Nd}(\mathrm{thf})$. Allerdings sind mehrere tert-Butyl-Gruppen in diesem Molekül so stark fehlgeordnet, daß eine eingehende Diskussion der Bindungslängen und -winkel von 3 nicht sinnvoll erscheint.

1.3 C. J. Burns, R. A. Andersen, J. Organomet. Chem. 325 (1987) 31.

${ }^{14)}$ M. F. Lappert, A. Singh, J. L. Atwoood, W. E. Hunter, J. Chem. Soc., Chem. Commun. 1981, 1191.

15) F. Benetollo, G. Bombicri, C. B. Castellani, W. Jahn, R. D. Fischer, Inorg. Chim. Acta 95 (1984) 17.

${ }^{161} \mathrm{Q}$. Shen, W. Chen, Y. Jin, C. Shan, Pure Appl. Chem. 60 (1988) 1251 und dort zit. Lit.

17) D. C. Bradley, J. S. Ghotra, F. A. Hart, J. Chem. Soc., Dalton Trans. 1973, 1021.

18) L. Syper, Rocz. Chem. 47 (1973) 433.

14) K. Rossmanith, Monatsh. Chem. 100 (1969) 1484.

2i) Weitere Einzelheiten zu den Kristallstrukturuntersuchungen können beim Fachinformationszentrum Karlsruhe, Gesellschaft für wissenschaftlich-technische Information $\mathrm{mbH}, \mathrm{D}-7514 \mathrm{Eg}$ genstein-Leopoldshafen 2, unter Angabe der Hinterlegungsnummer CSD-54720, der Autorennamen und des Zeitschriftenzitats angefordert werden.

$[246 / 90]$ 